\title{
AN ACYLASE FROM SHEWANELLA PUTREFACIENS PRESENTS A VIBRIO PARAHAEMOLYTICUS ACYLHOMOSERINE LACTONE-DEGRADING ACTIVITY AND EXHIBITS TEMPERATURE-, PH- AND METAL-DEPENDENCES
}

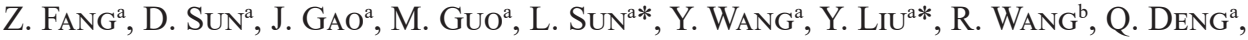 \\ D. XU ${ }^{\mathrm{a}}$ and R. GOONERATNE

\begin{abstract}
${ }^{a}$ College of Food Science and Technology, Guangdong Ocean University, Zhanjiang, 524048, China ${ }^{\mathrm{b}}$ College of Food Science and Engineering, Lingnan Normal University, Zhanjiang, 524048, China
\end{abstract} \\ ${ }^{\mathrm{c}}$ Department of Wine, Food and Molecular Biosciences, Lincoln University, Lincoln, Canterbury, 7647, \\ New Zealand
}

(Received: 4 February 2020; accepted: 9 July 2020)

Shewanella putrefaciens supernatant was found to increase the virulence factors of Vibrio parahaemolyticus by efficiently degrading its acylhomoserine lactone (AHL). To further reveal the regulation mechanism and its key degrading enzyme, a potential AHL-degrading enzyme acylase (Aac) from S. putrefaciens was cloned, and the influences of temperature, $\mathrm{pH}$, protein modifiers, and metals on Aac were tested. Aac was significantly influenced by temperature and $\mathrm{pH}$, and exhibited the highest AHL-degrading activity at temperatures of $37{ }^{\circ} \mathrm{C}$ and $\mathrm{pH}$ of $8 . \mathrm{Mg}^{2+}$ and $\mathrm{Fe}^{2+}$ can further increase the AHL-degrading activity. $10 \mathrm{mM}$ EDTA inhibited its activity possibly by chelating the co-factors (metals) required for Aac activity. Tryptophan and arginine were identified as key components for Aac activity that are critical to its AHL-degrading activity. This study provides useful information on Aac and for $V$. parahaemolyticus control.

Keywords: acylase, AHL-degrading enzyme, acylhomoserine lactone, Shewanella putrefaciens, Vibrio parahaemolyticus

As an occasional foodborne pathogen in seafood, Vibrio parahaemolyticus has been responsible for gastroenteritis outbreaks worldwide. Shewanella putrefaciens coexists with $V$. parahaemolyticus and increases virulence factors of V. parahaemolyticus in shrimp (WRIGHT et al., 2016; FANG et al., 2018). S. putrefaciens is a major food spoilage bacterium in seafood, and exhibits a higher resistance to antibiotics and metals (KANG \& So, 2016), but rarely implicated as a cause of human disease (STEINBERG \& BURD, 2015). Ambient temperature, $\mathrm{pH}$, and metals are also virulence factors of vibrio pathogens (GoDE-Potratz et al., 2010; Gode, 2011; Kimes et al., 2012; Gutierrez et al., 2013). However, its mechanism of regulating the virulence factors of $V$. parahaemolyticus is not well understood. Quorum sensing (QS) is a bacterial communication system that regulates virulence factors and antibiotic effects via synthesis of autoinducer molecules (HAMmER \& BASSLER, 2003; SHIH \& HUANG, 2002). Vibrio harveyi AHL autoinducer is degraded by bacterial enrichment cultures of shrimp (TinH et al., 2007). The virulence gene hlyA is negatively regulated by the QS system in Vibrio cholerae (Tsou \& ZHU, 2010). It is thought that QS signal degrading enzymes can alter the virulence factors.

* To whom correspondence should be addressed.

Phone: +86 759 2396027; fax: +86759 2396027; e-mails: suncamt@126.com (L. Sun); liuyingxk@sina.com (Y. LIU) 
In a previous study, we reported that the $S$. putrefaciens supernatant exhibited a significant degradation ability of QS signal AHL of $V$. parahaemolyticus (FANG et al., 2018). Variovorax paradoxus can also utilise AHLs as energy sources for growth (LEADBETTER \& GrEenBERG, 2000). Ralstonia sp. aiiD was identified as a potent AHL acylase (LIN et al., 2003). Shewanella oneidensis AHL-acylase (Aac) is an aiiD homolog and contains a highly conserved Ntn_hydrolase, which has a structure similar to the common of $\beta$-lactamases superfamily (KIM et al., 2006). It is also reported to markedly degrade the AHL production of Vibrio anguillarum (MoroHoshi et al., 2008). AHL acylase of Shewanella algae belongs to the penicillin acylase family, which can confer $S$. algae resistant to penicillins and other $\beta$-lactam antibiotics (GHANEIMOTLAGH et al., 2019). Zinc and other metal-binding motifs are common in the Ntn_hydrolase structure of (Melino et al., 1998; IGNATOVA et al., 2005). In this study, we investigated the Aac properties by testing the effects of temperature, $\mathrm{pH}$, modifiers, and metals on its AHL degrading ability, to further reveal the mechanisms regulating the virulence factors of $V$. parahaemolyticus by $S$. putrefaciens in spoiled shrimp.

\section{Materials and methods}

\subsection{Bacterial strains, plasmids, and growth conditions}

The V. parahaemolyticus strain ATCC33847 (from the China Committee for Culture Collection of Microorganisms), Escherichia coli BL21, and DH5 $\alpha$ (from our lab) carrying plasmids pET28a $(+)\left(\mathrm{kan}^{\mathrm{r}}\right.$, from our lab) were grown in LB culture $\left(10 \mathrm{~g} \mathrm{l}^{-1}\right.$ yeast extract, 10 $\mathrm{g} \mathrm{l}^{-1}$ tryptone, and $\left.5 \mathrm{~g} \mathrm{l}^{-1} \mathrm{NaCl}\right)$ with or without Kanamycin $\left(50 \mu \mathrm{g} \mathrm{ml}^{-1}\right)$ at $37{ }^{\circ} \mathrm{C}$, unless specifically noted otherwise. Kanamycin (USP Grade,), yeast extract (FMB Grade), tryptone (FMB Grade), $\mathrm{NaCl}$ (purity $\geq 99.5 \%$ ), $\mathrm{MgCl}_{2}$ (purity $\geq 99.0 \%$ ), and metals and reagents were purchased from Sangon Biotech Co., Ltd (Shanghai, China).

\subsection{Synthesis and expression of the aac gene in plasmid pET28a}

The $S$. putrefaciens aac gene was synthesised by Sangon Biotech Co., Ltd (Shanghai, China) according to the sequence of the $a a c$ gene from $S$. putrefaciens strain in the database (NCBI Accession Number NC_009438). The aac DNA fragment and plasmid pET28a were digested with BamHI and Hind III, and the PCR products were purified with gel electrophoresis and ligated by T4 ligase according to FANG and co-workers (2015).

The E.coli BL21 harbouring recombinant pET28a-aac was grown in LB culture with Kanamycin $\left(50 \mu \mathrm{g} \mathrm{ml}^{-1}\right)$ at $37^{\circ} \mathrm{C}$ for $24 \mathrm{~h}$, transferred to a new LB culture, and induced with $1 \mathrm{mM}$ isopropylthio-galactoside (IPTG, purity $\geq 99.0 \%$, Sangon Biotech Shanghai, China) for $6 \mathrm{~h}$. The recombinant protein was harvested and purified according to MoROHOSHI and co-workers (2008). Briefly, the cultured supernatants were ultrafiltered with an Amicon Ultra-15 membrane (10 kDa, Millipore-Sigma, USA) at $3000 \mathrm{~g}$ at $4{ }^{\circ} \mathrm{C}$ for $2 \mathrm{~h}$, and the condensed supernatant was diluted with pre-cooled LB culture to $1: 100(\mathrm{v}: \mathrm{v})\left(10 \mu \mathrm{g} \mathrm{ml}^{-1}\right)$ for further analysis.

\subsection{Determination of $A H L$-degrading rates}

V. parahaemolyticus AHL was prepared as reported by FANG and co-workers (2018). AHL was added to a $500 \mu \mathrm{l}$ suspension of E.coli BL21 harbouring pET28a-aac containing $4 \mathrm{mM}$ protein modifier or $5 \mathrm{mM}$ metal ions or EDTA to react at $25,30,37,45,50$, and $55^{\circ} \mathrm{C}$ for 30 
min as described by MoROHOSHI and co-workers (2008), at a final concentration of $5 \mu \mathrm{g} \mathrm{ml}^{-1}$. The residual AHL contents were determined by liquid chromatography linked to tandem mass spectrometry (LC-MS/MS; Tandem Quadrupole LCMS-8030 (Shimadzu, Japan)) as described by FANG and co-workers (2018). For AHL analysis, the injection volume was set at $10.0 \mu \mathrm{l}$, and eluent A was methanol and eluent B was water at a flow rate $0.3 \mathrm{ml} \mathrm{min}^{-1}$. The elution gradient started with $30 \%$ of eluent $\mathrm{A}$, was raised to $90 \%$ in a 4 min gradient, and then was set back to the initial conditions after 3 min. For mass spectra in ESI source, the block and desolvation temperatures were $250^{\circ} \mathrm{C}$ and $400^{\circ} \mathrm{C}$, respectively, desolvation gas flow rate was $15.01 \mathrm{~min}^{-1}$ and capillary voltage was $4.5 \mathrm{kV}$. The ratios of LC-MS/MS peak areas of the analytes to an internal standard were measured, and the AHL samples treated without Aac supernatants were set as blank controls $(0 \%)$. The data were adjusted by subtraction from the corresponding blank controls. Each experiment was performed at least 3 times.

\subsection{Statistical analysis}

Statistical analysis was performed with ANOVA and Duncan's multiple range test using SPSS version 19.0. The results were compared and the statistical significance $P$ value was set at $<0.05$.
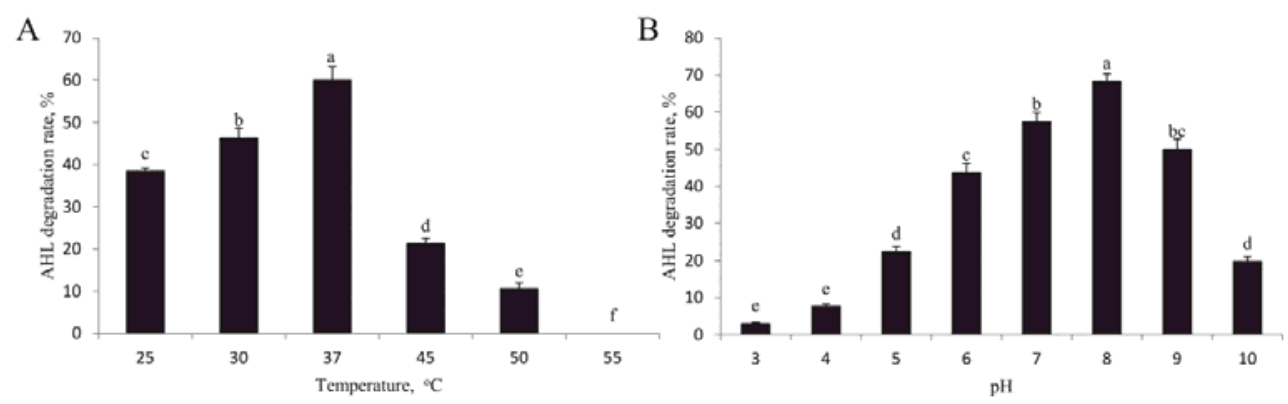

Fig. 1. Effects of temperature and $\mathrm{pH}$ on AHL-degrading activity of S. putrefaciens lactonase enzyme Aac. 500 $\mu 1$ supernatant of BL21 harbouring pET28a-aac was mixed with $100 \mu \mathrm{AHL}$, incubated at 25, 30, 37, 45, 50, and $55^{\circ} \mathrm{C}(\mathrm{A})$ or at $\mathrm{pH} 3,4,5,6,7,8,9$, and 10 (B) for $30 \mathrm{~min}$. the AHL contents were measured with LC-MS/MS. The data represent the means of three independent experiments. Error bars represent mean standard deviations. Different superscript letters indicate significant differences at $\mathrm{P}<0.05$ according to Duncan's test.

\section{Results and discussion}

\subsection{Effects of temperature and $p H$ on $\mathrm{V}$. parahaemolyticus $A H L$-degrading activity of S. putrefaciens $A a c$}

S. putrefaciens regulates V. parahaemolyticus virulence factors through its AHL-degrading ability, and the temperature influences this regulation (FANG et al., 2018). To detect the potential AHL degrading activity of $S$. putrefaciens Aac and its other properties, the AHLdegrading rates of $S$. putrefaciens Aac and the effect of temperature on its activity were determined. V. parahaemolyticus AHL was mixed with $500 \mu \mathrm{l}$ of Aac solution to react at different temperatures for $30 \mathrm{~min}$. S. putrefaciens Aac exhibited a higher degrading activity at $37{ }^{\circ} \mathrm{C}>30^{\circ} \mathrm{C}>25^{\circ} \mathrm{C}$ with a clear positive temperature-dependent effect. However, when the temperature was $>37^{\circ} \mathrm{C}$, the AHLs degrading activity of Aac decreased. Thus, the highest 
AHL-degrading activity was at $37^{\circ} \mathrm{C}$. This is consistent with our previous findings that $37^{\circ} \mathrm{C}$ was the optimal temperature for maximal $S$. putrefaciens activity to enhance the $V$. parahaemolyticus virulence factors (FANG et al., 2018).

Next, the effect of $\mathrm{pH}$ on Aac in AHL-degrading was analysed. For this, AHL was mixed with Aac solution to react at different $\mathrm{pHs}(0.1 \mathrm{mM}$ Tris- $\mathrm{HCl}, \mathrm{pH}=3$ to 10$)$ for $30 \mathrm{~min}$. At $\mathrm{pHs}<8$, Aac exhibited a positive $\mathrm{pH}$-dependent-effect on AHL degradation. At $\mathrm{pHs}>8$, the degrading activity of Aac was suppressed. The optimal pH for Aac was 8. S. putrefaciens Aac showed a better pH-stability compared to that of Bacillus sp. (WANG et al., 2004).

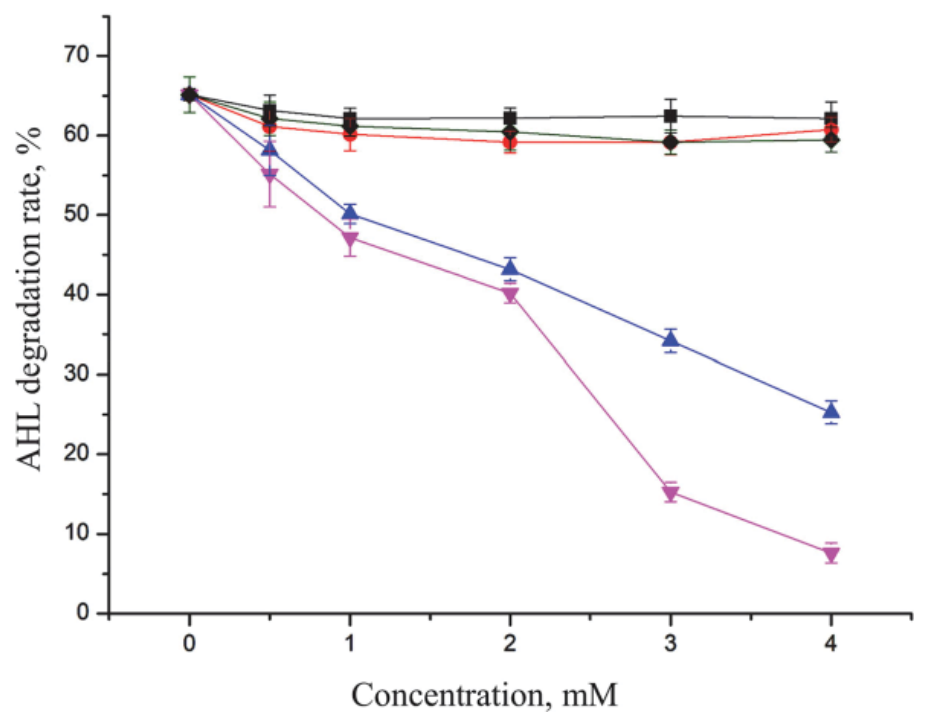

Fig. 2. Effects of protein modifiers on AHL-degrading activity of S. putrefaciens lactonase enzyme Aac. The mixture of Aac and AHL was incubated with or without indicated concentrations of DTT, BrAc, Acac, NBS, and $\mathrm{PMSF}$ at $37^{\circ} \mathrm{C}$ for $30 \mathrm{~min}$. The AHL contents were measured with LC-MS/MS. The data represent the means of three independent experiments. Error bars represent mean standard deviations.

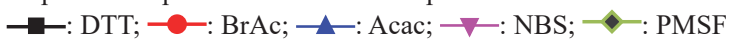

\subsection{Effects of protein modifiers on $\mathrm{S}$. putrefaciens Aac}

The Aac activity is usually ascribed to its protein structure. Ntn hydrolase is a key conserved structural domain of Aac and is also found in ATP-dependent proteases (KIM et al., 2000). Such structural domains are usually rich in arginine, serine, lysine or histidine residues. Besides, disulphide bonds offer structural stability but can be broken by dithiothreitol (DTT). Arginine residues are modified by acetylacetone (Acac), which is used to confirm the presence of arginine residues in proteins (DiKLER et al., 2015). BrAc was used to analyse the histidine active site residues (MORGAN \& MULLEREBERHARD, 1976), and N-bromo succinimide (NBS) was used to analyse tryptophan based active site residues (TeNG et al., 2006). 5-methylphenazinium methyl sulphate (PMSF), as a routine protease inhibitor, was used to modify serine based active site residues. In order to analyse and confirm the Aac enzymatic properties and key active site residues, DTT, BrAc, PMSF, NBS, and Acac were used to test their effects on AHL degrading activity of Aac. Compared to DTT, BrAc, and PMSF, addition of $1 \mathrm{mM}$ or higher doses of NBS and Acac significantly reduced the AHL degrading activity 
of Aac (Fig. 2), suggesting that tryptophan and arginine residues are the key active sites of Aac. The results showed that Aac was significantly inhibited by NBS and Acac, which is highly specific for tryptophan and arginine residues (TENG et al., 2006; DiKLER et al., 2015). AHL acylase and GL-7-ACA acylase both belong to family of $\beta$-lactam acylases with a conserved Ntn hydrolase (KIM et al., 2000; KIM et al., 2006). In previous reports, tryptophan and arginine were also found to be involved in the substrate binding and catalytic activity of GL-7-ACA acylase in Pseudomonas sp. (LEE et al., 2000). These results suggest that tryptophan and arginine residues play important roles in the AHL degradation activity of Aac.

\subsection{Effects of metals on S. putrefaciens Aac}

Most beta-lactam acylases are metal-dependent enzymes. Zinc-binding motif is conserved in $\beta$-lactam acylases family, and is influenced by zinc (MeLINo et al., 1998). As an aiiD homolog, $S$. putrefaciens Aac may have a common structure to $\beta$-lactamases superfamily (KIm et al., 2006). In order to further analyse the properties of Aac, its metal dependence was tested. 5 $\mathrm{mM} \mathrm{Mg}{ }^{2+}, \mathrm{Fe}^{2+}, \mathrm{Ca}^{2+}, \mathrm{Cu}^{2+}, \mathrm{Zn}^{2+}, \mathrm{Ba}^{2+}$, and $\mathrm{Mn}^{2+}$ were added to Aac and AHL mixed solution to react at $37{ }^{\circ} \mathrm{C}$ for $30 \mathrm{~min}$ (Fig. $3 \mathrm{~A}$ ). $5 \mathrm{mM} \mathrm{Mg}^{2+}$ and $\mathrm{Fe}^{2+}$ significantly enhanced the AHLdegrading activity of Aac compared to the control (without metals). This explains why $\mathrm{Fe}^{2+}$ could enhance the $V$. anguillarum virulence in Japanese eels and ayu (NAKAI et al., 1987). However, $5 \mathrm{mM} \mathrm{Ca}{ }^{2+}, \mathrm{Cu}^{2+}, \mathrm{Ba}^{2+}$, and $\mathrm{Mn}^{2+}$ significantly suppressed the AHL-degrading activity of Aac. The effect of $\mathrm{Zn}^{2+}$ on Aac was slightly negative but not obvious. However, a significant decrease in AHL-degrading activity was observed in Aac following the addition of $10 \mathrm{mM}$ or higher EDTA (a metal chelator) concentration (Fig. 3B). In other bacteria, some typical acylase activities have been found to be increased by $\mathrm{Mg}^{2+}$ (Zou et al., 2016). The lactonases from Burkholderia sp. and Bacillus sp. were inhibited by $\mathrm{Cu}^{2+}$ and $\mathrm{Zn}^{2+}$ but not by ion-chelating EDTA (MochIZUKI, 2001), which did not explain that $\mathrm{Zn}^{2+}$ is required for catalytic activity of lactonase with a zinc-binding motif (CROWDER et al., 1997). When the concentration of ion-chelating reagent EDTA was increased to remove the residual $\mathrm{Zn}^{2+}$ from Aac, the AHL degradation activity significantly decreased. These results together indicate that metals are also required for the AHL degrading activity of Aac, confirming that metals increase $V$. parahaemolyticus virulence factors by enhancing the Aac activity of $S$. putrefaciens or other symbiotic bacteria in shrimp.
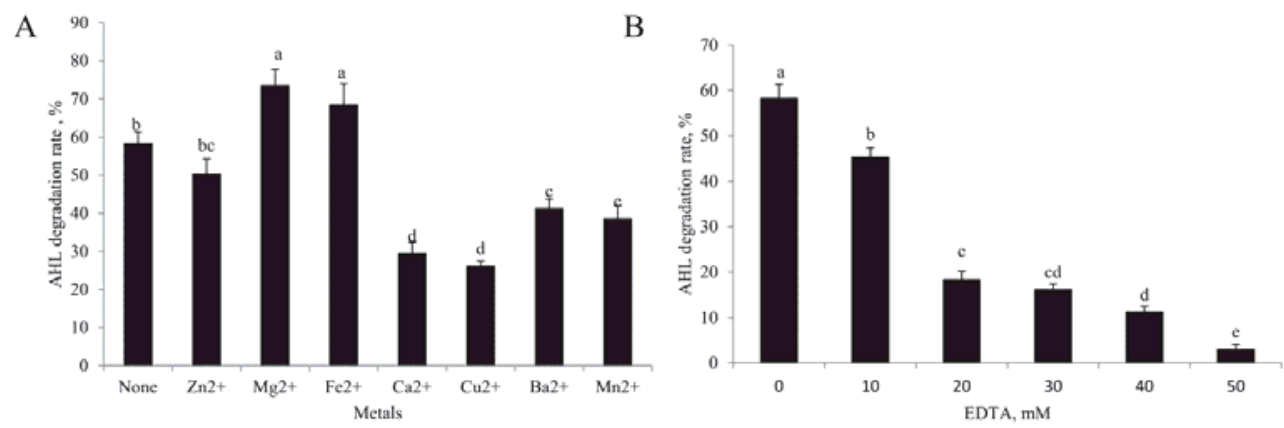

Fig. 3. Effects of metals on AHLs degrading activity of $S$. putrefaciens Aac. The mixture of Aac and AHL was incubated with or without $5 \mathrm{mM} \mathrm{Mg}{ }^{2+}, \mathrm{Fe}^{2+}, \mathrm{Ca}^{2+}, \mathrm{Cu}^{2+}, \mathrm{Zn}^{2+}, \mathrm{Ba}^{2+}$, and $\mathrm{Mn}^{2+}$ (A) or indicated concentration of EDTA (B) at $37^{\circ} \mathrm{C}$ for $30 \mathrm{~min}$. The AHL contents were measured with LC-MS/MS. The data represent the means of three independent experiments. Error bars represent mean standard deviations. Different superscript letters indicate significant differences at $\mathrm{P}<0.05$ according to Duncan's test. 


\section{Conclusions}

In this study, we cloned a putative AHL acylase gene aac from S. putrefaciens and found that Aac could degrade QS autoinducer molecule AHL and increase virulence factors of $V$. parahaemolyticus. S. putrefaciens Aac was sensitive to environmental temperature, $\mathrm{pH}$, and metals. It is noteworthy that Acac, NBS, and EDTA significantly impaired Aac. The key amino acid residues, such as tryptophan and arginine, and metals also contribute to its AHLdegrading activity, and $\mathrm{Mg}^{2+}$ and $\mathrm{Fe}^{2+}$ can further enhance it. These findings further confirmed the AHL degradation activity of $S$. putrefaciens Aac as an AHL acylase. More importantly, the Aac contributes to the increase in V. parahaemolyticus virulence factors. Lowering the activity of Aac using inhibitors could be an effective strategy to control the $V$. parahaemolyticus virulence in shrimp preservation.

This work was supported by funds from the National Natural Science Foundation of China (grant number 31371746, 31371777.) and Higher Educational Cultivation Program for Major Scientific Research Projects of Guangdong Ocean University (grant number GDOU2013050205, 2014050203).

\section{References}

Crowder, M.W., Maiti, M.K., Banovic, L. \& Makaroff, CA. (1997): Glyoxalase II from A. thaliana requires $\mathrm{Zn}(\mathrm{II})$ for catalytic activity. FEBS Lett., 418(3), 351-354.

DiKLER, S., KelLY, J.W. \& RuSSELL, D.H. (2015): Improving mass spectrometric sequencing of arginine-containing peptides by derivatization with acetylacetone. J. Mass Spectrom., 32(12), 1337-1349.

Fang, Z., Kuang, X., Zhang, Y., Shi, P. \& Huang, Z. (2015): A novel HAC1-based dual-luciferase reporter vector for detecting endoplasmic reticulum stress and unfolded protein response in yeast Saccharomyces cerevisiae. Plasmid, 79, 48-53.

Fang, Z., Sun, D., Li, C., Sun, L., Wang, Y., Guo, M., ... \& Liu, Y. (2018): Regulatory effects of Shewanella putrefaciens isolated from shrimp Penaeus orientalis on the virulence factors of Vibrio parahaemolyticus and evaluation of the role of quorum sensing in virulence factors regulation. FEMS Microbiol. Ecol., 94(7), fiy097.

Ghaneimotlagh, R., Mohammadian, T., Gharibi, D., Menanteauledouble, S., Mahmoudi, E., ... \& Elmatbouli, M. (2019): Quorum quenching properties and probiotic potentials of intestinal associated bacteria in Asian sea bass Lates calcarifer. Mar. Drugs, 18(1), 23.

GoDE, C.J. (2011): Vibrio parahaemolyticus responds to growth on a surface by initiating a program of gene control that is regulated by calcium, iron, and quorum sensing. (PhD Thesis), University of Iowa, Iowa, US. 208 pages.

Gode-Potratz, C.J., Chodur, D.M. \& Mccarter, L.L. (2010): Calcium and iron regulate swarming and type III secretion in Vibrio parahaemolyticus. J. Bacteriol., 192(22), 6025.

Gutierrez West, C.K., KLein, S.L. \& Lovell, C.R. (2013): High frequency of virulence factor genes tdh, trh, and th in Vibrio parahaemolyticus strains isolated from a pristine estuary. Appl. Environ. Microb., 79(7), 22472252.

HAMmer, B.K. \& BASSLER, B.L. (2003): Quorum sensing controls biofilm formation in Vibrio cholerae. Mol. Microbiol., 50(1), 101-104.

Ignatova, Z., Wischnewski, F., Notbohm, H. \& Kasche, V. (2005): Pro-sequence and $\mathrm{Ca}^{2+}$-binding: Implications for folding and maturation of ntn-hydrolase penicillin amidase from E. coli. J. Mol. Biol., 348(4), 999-1014.

KANG, C. \& So, J. (2016): Antibiotic and heavy metal resistance in Shewanella putrefaciens strains isolated from shellfishes collected from West Sea, Korea. Mar. Pollut. Bull., 112(1), 111-116.

Kim, J.K., YAng, I.S., Shin, H.J., Cho, K.J., Ryu, E.K., Kıм, S.H., . . \& KIm, K. H. (2006): Insight into autoproteolytic activation from the structure of cephalosporin acylase: A protein with two proteolytic chemistries. P. Natl. Acad. Sci. USA., 103(6), 1732-1737.

Kim, Y., Yoon, K.-H., Khang, Y., Turley, S. \& Hol, W.G. (2000): The 2.0 A crystal structure of cephalosporin acylase. Structure, 8(10), 1059-1068. 
Kimes, N.E., Grim, C.J., Johnson, W.R., Hasan, N.A., Tall, B.D., Kothary, M.H., . . . \& Morris, P.J. (2012): Temperature regulation of virulence factors in the pathogen Vibrio coralliilyticus. ISME J, 6(4), 835-846.

LeAdbetter, J.R. \& GreenberG, E.P. (2000): Metabolism of acyl-homoserine lactone quorum-sensing signals by Variovorax paradoxus. J. Bacteriol., 182(24), 6921-6926.

LeE, Y.S., KIM, H.W., KANG, B.L. \& PARK, S.S. (2000): Involvement of arginine and tryptophan residues in catalytic activity of glutaryl 7-aminocephalosporanic acid acylase from Pseudomonas sp. strain GK16. Biochim. Biophys. Acta, 1523(1), 123-127.

Lin, Y., Xu, J., Hu, J., Wang, L., Ong, S.L., Leadbetter, J.R., Zhang, L.H. (2003): Acyl-homoserine lactone acylase from Ralstonia strain XJ12B represents a novel and potent class of quorum-quenching enzymes. Mol. Microbiol., 47(3), 849-860.

Melino, S., Capo, C., Dragani, B., Aceto, A. \& Petruzzelli, R. (1998): A zinc-binding motif conserved in glyoxalase II, beta-lactamase and arylsulfatases. Trends Biochem. Sci, 23(10), 381-382.

MochizUKI, K. (2001): Purification and characterization of a lactonase from Burkholderia sp. R-711, that hydrolyzes (R)-5-oxo-2-tetrahydrofurancar-boxylic acid. Arch. Microbiol., 175(6), 430-434.

Morgan, W.T. \& Mullereberhard, U. (1976): Chemical modification of histidine residues of rabbit hemopexin. Arch. Biochem. Biophys., 176(2), 431-441.

Morohoshi, T., Nakazawa, S., Ebata, A., Kato, N. \& Ikeda, T. (2008): Identification and characterization of $\mathrm{N}$-acylhomoserine lactone-acylase from the fish intestinal Shewanella sp. strain MIB015. Biosci., Biotech., Bioch., 72(7), 1887-1893.

Nakai, T., Kanno, T., Cruz, E.R. \& Muroga, K. (1987): The effects of iron compounds on the virulence of Vibrio anguillarum in Japanese eels and ayu. Fish Pathol., 22(4), 185-189.

Shin, P.C. \& Huang, C.T. (2002): Effects of quorum-sensing deficiency on Pseudomonas aeruginosa biofilm formation and antibiotic resistance. J. Antimicrob. Chemoth., 49(2), 309-314.

SteinberG, J.P. \& Burd, E.M. (2015): 238 - Other Gram-negative and Gram-variable bacilli. -in: BennetT, J.E., Dolin, R. \& Blaser, M.J. (Eds): Mandell, Douglas, and Bennett's principles and practice of infectious diseases $8^{\text {th }}$. Saunders Elsevier, Philadelphia. pp. 2667-2683

Teng, L.R., Fan, H., Zhang, Y.Y., Qi, Y.U., Huang, Y.F. \& LiU, L.Y. (2006): Chemical modification and fluorescence spectrum of tryptophan residues in pullulanase. Chem. Res. Chinese U., 22(01), 69-72.

Tinh, N.T.N., Gunasekara, R.A.Y.S., Boon, N., Dierckens, K., Sorgeloos, P. \& Bossier, P. (2007): N-acyl homoserine lactone-degrading microbial enrichment cultures isolated from Penaeus vannamei shrimp gut and their probiotic properties in Brachionus plicatilis cultures. FEMS Microbiol. Ecol., 62(1), 45-53.

Tsou, A.M. \& ZHU, J. (2010): Quorum sensing negatively regulates hemolysin transcriptionally and posttranslationally in Vibrio cholerae. Infect. Immun., 78(1), 461-467.

Wang, L.H., Weng, L.X., Dong, Y.H. \& Zhang, L.H. (2004): Specificity and enzyme kinetics of the quorumquenching N-acyl homoserine lactone lactonase (AHL-lactonase). J. Biol. Chem, 279(14), 13645-13651.

Wright, M.H., Matthews, B., Arnold, M.S.J., Greene, A.C. \& Cock, I.E. (2016): The prevention of fish spoilage by high antioxidant Australian culinary plants: Shewanella putrefaciens growth inhibition. Int. J. Food Sci. Tech., 51(3), 801-813.

Zou, C., Duan, X. \& Wu, J. (2016): Magnesium ions increase the activity of Bacillus deramificans pullulanase expressed by Brevibacillus choshinensis. Appl. Microbiol. Biot., 100(16), 7115-7123. 